\title{
A Survey of Resource Allocation Techniques for Cellular Network's Operation in the Unlicensed Band
}

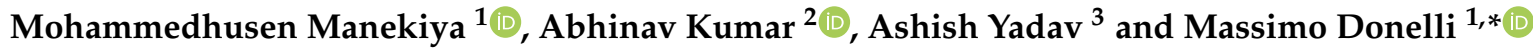 \\ 1 Department of Information Engineering and Computer Science, University of Trento, 38123 Trento, Italy; \\ m.manekiya@unitn.it \\ 2 Department of Electrical Engineering, Indian Institute of Technology Hyderabad, Telangana 502285, India; \\ abhinavkumar@iith.ac.in \\ 3 Department of Electrical and Computer Engineering, Tandon School of Engineering, New York University, \\ Brooklyn, NY 11201, USA; ay1000@nyu.edu \\ * Correspondence: massimo.donelli@unitn.it; Tel.: +39-3297-00-4115
}

Received: 1 August 2020; Accepted: 27 August 2020; Published: 7 September 2020

check for updates

\begin{abstract}
With an ever increasing demand for data, better and efficient spectrum operation has become crucial in cellular networks. In this paper, we present a detailed survey of various resource allocation schemes that have been considered for the cellular network's operation in the unlicensed spectrum. The key channel access mechanisms for cellular network's operation in the unlicensed bands are discussed. The various channel selection techniques are explored and their operation explained. The prime issue of fairness between cellular and Wi-Fi networks is discussed, along with suitable resource allocation techniques that help in achieving this fairness. We analyze the coverage, capacity, and impact of coordination in LTE-U systems. Furthermore, we study and discuss the impact and discussed the impact of various traffic type, environments, latency, handover, and scenarios on LTE-U's performance. The new upcoming 5G New Radio and MulteFire is briefly described along with some of the critical aspects of LTE-U which require further research.
\end{abstract}

Keywords: CSAT; LAA-LBT; LTE-U resource allocation; operations in unlicensed band

\section{Introduction}

The demand for data in cellular networks has increased exponentially in the past few decades. To meet this ever-increasing demand, a several-fold increase in spectrum is required [1]. However, the cost of the licensed spectrum has become exorbitantly high. Thus, cellular network operators are continuously looking for technological solutions to efficiently operate their networks with minimum cost for spectrum. This has resulted in the rapid adoption of Long-Term Evolution (LTE) based cellular networks. However, most of the available licensed spectrum in lower than $6 \mathrm{GHz}$ bands has already been allocated. The co-existence of multiple access technologies in the $2.4 \mathrm{GHz}$ unlicensed spectrum termed as the industrial, scientific and medical (ISM) band makes it unsuitable for operation of LTE [2]. Therefore, based on the availability across the globe, the $5 \mathrm{GHz}$ unlicensed national information infrastructure (U-NII) band can be used for the operation of LTE based cellular networks with suitable modifications.

The unlicensed spectrum can be used by LTE in broadly three ways. One way is to offload LTE traffic to Wi-Fi in the unlicensed spectrum [3]. A second is the integration of this unlicensed band with the licensed spectrum and utilization of LTE air-interface with minimum changes (also referred to as LTE-U) [4]. A third way is license assisted access with a listen before talk (LAA-LBT) feature potentially in LTE Release 13 (Rel-13) [5]. The benefits of using LTE-U or LAA-LBT rather than a hybrid solution of LTE and Wi-Fi are the subject of ongoing discussions within the research community [6] 
and is beyond the scope of this article. In this article, we focus on the second and the third, i.e., the LTE-U and LAA-LBT, respectively, as they have become promising technologies to offer increased data rates without increase in the cost of spectrum.

Traditionally, the $5 \mathrm{GHz}$ unlicensed band has been used for Wi-Fi, RADAR, and other applications [2]. Given the unlicensed nature, several users can simultaneously operate in this band without any coordination. Thus, the persistence of transmissions of LTE-U can significantly degrade the performance of incumbent devices in the unlicensed band which employ carrier sense multiple access (CSMA) as medium access control (MAC) protocol [7]. The motivation of this article is to explore in-depth the resource allocation techniques proposed in the literature for channel access mechanisms and channel selection schemes for LTE-U and LAA-LBT that ensure fair coexistence with existing devices. We also study the impact of coordination, traffic type, and the environment on the capacity, coverage, and latency of LTE-U and LAA-LBT using the resource allocation techniques co-existing with Wi-Fi. Furthermore, we examine in detail the key issues in LTE-U and LAA-LBT that should be addressed. We present possible future works that can help network regulators and cellular operators in adoption of best policies for LTE's operation in the unlicensed band while keeping in mind the interest of existing Wi-Fi users.

For the two different potential technologies for LTE's operation in the unlicensed band, the available spectrum and the channel access mechanisms in the unlicensed spectrum are region specific. For example, the different bands and their functioning in the United States (US) is explained in detail in [2]. The unlicensed spectrum layout for some of the key cellular markets have been studied in [8], while [9] presents a detailed insight of all the sub-bands in the $5 \mathrm{GHz}$ band for the major markets. The two key region specific channel access mechanisms for LTE's operation in the unlicensed band that are globally under consideration are carrier sensing adaptive transmission (CSAT) and LAA-LBT. For non-LBT markets such as South Africa and India, CSAT is proposed to be used with Rel-10 carrier aggregation protocols for co-channel existence. In case of LBT markets like Japan and Europe, Rel-13 with LAA-LBT will be used [5]. In Figure 1, some of the key cellular markets with the corresponding channel access mechanism for LTE's operation in the unlicensed band are presented [5,10]. It is observed from Figure 1 that a significant proportion of the cellular network regulators across the globe are planning/conducting trials with both the channel access mechanisms for LTE's operation in the unlicensed band. Furthermore, a sizable proportion of the global markets have not started experimenting with either of the technologies. Hence, this article is even more relevant, as the presented results can be used by the regulators and network operators to make suitable decisions in the context of LTE's operation in the unlicensed band. Most regulations put limits on transmission powers in the unlicensed bands. For instance, for the lower $5 \mathrm{GHz}$ band, the maximum transmission power in Europe is $23 \mathrm{dBm}$ EIRP. As a result of the transmission power limits, LAA will generally be more suited for small cell deployments.

The organization of this paper is as follows. The introduction and related works are covered in Sections 1 and 2, respectively. In Section 3, we present the channel access mechanisms for LTE-U. The channel selection techniques for LTE-U are discussed in Section 4. In Section 5, the fairness of these mechanisms and techniques in terms of the scaling with the users, operators, and access points is evaluated. The coverage, capacity, and coordination in the context of LTE-U are analyzed in Section 6. The LTE-'s performance evaluation in diverse traffic type, environments, and scenarios is studied in Section 7. In Section 8, latency and handover scenarios for LTE-U are analyzed. The upcoming 5G New Radio and MulteFire technologies are discussed in Sections 9 and 10, respectively. The open issues in LTE-U are discussed in Section 11, followed by some concluding remarks in Section 12. 


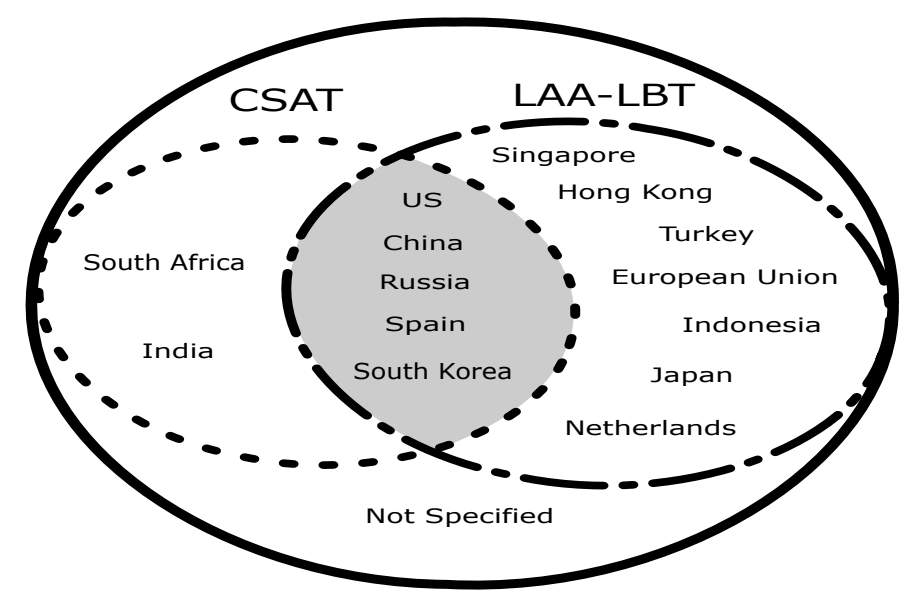

Figure 1. A region wise classification of trials and deployments of various channel access mechanisms for LTE's operation in the unlicensed band.

\section{Related Work}

An in-depth survey of the coexistence of LTE-LAA and Wi-Fi on the $5 \mathrm{GHz}$ unlicensed band has been presented in [11]. In [11], various coexistence related features of LTE-LAA and Wi-Fi have been discussed in detail. Furthermore, several deployment scenarios and scenario oriented decision-making along with possible research directions have been presented. However, fair coexistence of LTE-LAA (or LTE-U) and Wi-Fi is dependent on the resource allocation schemes used in LTE-LAA (or LTE-U) and Wi-Fi. Thus, in this work, we present a detailed survey of channel selection and channel access mechanisms for both LTE-LAA and LTE-U from the perspective of resource allocation.

In [12], an overview of the challenges and requirements of the fronthaul technology in LTE-U based small cells (SCs) in 5G networks has been presented. Furthermore, the advantages and challenges for various candidate fronthaul technologies have been discussed. However, the important issues of fairness, coexistence, and handover have not been discussed in [12]. Similarly, in [13], the impact of several design parameters of the LAA-LBT evaluation framework on the channel access opportunities of LAA and its coexistence have been studied, for both single-channel as well as multi-channel operation. Key design specification with different channel access methods for coexistence framework of LAA and Wi-Fi based on release 13 have been presented in [13]. However, suitable resource allocation techniques to optimize the performance of LAA-LBT and Wi-Fi have not been discussed.

In [14], an overview of the Rel-13 LAA technology including motivation, use cases, LTE enhancements for enabling the unlicensed band operation, and the coexistence evaluation results contributed by 3GPP participants have been provided. Performance results for the LAA-LBT coexistence mechanism have been presented. However, coverage, capacity, or fairness with respect to number of users, access points (APs), and SC have not been discussed. In [15], an overview of various technical issues like coexistence among the different operators, different RAT, handover between RAT and LTE-U, traffic balancing, and traffic loading in Wi-Fi mechanism have been discussed. However, issues from real-time hardware implementation and analysis of the LTE-U and LAA-LBT from a resource allocation perspective have not been included.

Unlike the existing surveys that are targeted only for coexistence of LTE and $\mathrm{Wi}-\mathrm{Fi}$, this paper focuses on the complete study of resource allocation techniques for LAA-LBT and LTE-U including game theory and machine learning for fair coexistence. We have also presented a summary on different traffic model, environments and performance evaluation of coverage, capacity and coordination for both uplink and downlink. Furthermore, we have discussed the real-time hardware implementation for LTE's operation in the unlicensed band. Additionally, we have explained some open issues and solutions for resource allocation in CSAT and LAA-LBT. 


\section{Channel Access Mechanisms}

The distributed coordination function (DCF) is the basic channel access mechanism for Wi-Fi devices operating in the $5 \mathrm{GHz}$ band [16]. In DCF, the Wi-Fi device performs an initial clear channel assessment for a pre-determined time (called as arbitration inter-frame spacing (AIFS) time of at least $34 \mu \mathrm{s})$ and transmits if the channel is idle. In case the channel is busy, the Wi-Fi device backs off for a random number of time slots from a pre-determined contention window, and then transmits based on availability of the channel. A successful transmission is followed by an acknowledgement (ACK). The received ACK is used to update the contention window. This contention window update requires knowledge of immediate ACK. The DCF cannot be directly used in the presence of delayed ACKs. However, the ACKs are typically delayed in LTE. Thus, for regions like Japan and Europe that have requirements for supporting LBT at the milliseconds time scale, LAA-LBT based channel access mechanisms have been proposed as follows.

\subsection{License Assisted Access with Listen before Talk (LAA-LBT)}

The commercial availability of LAA was first discussed in [5]. The LAA requires discontinuous transmission on a carrier with limited maximum transmission duration. The channel selection for LAA devices should select carriers with low interference and is required for radar avoidance in certain regions/bands (discussed in detail in next section). Transmit power control is another regulatory requirement in many regions that requires the transmitting LAA device to reduce the transmit power compared to the maximum nominal transmit power [17]. The LAA utilizes carrier aggregation such that the secondary carrier comprises of the unlicensed spectrum in the $5 \mathrm{GHz}$ U-NII bands and the primary carrier is the licensed spectrum [7].

The LAA based systems are required to perform LBT that includes a clear channel assessment (CCA) before accessing the channel using energy detection for a pre-determined time $(20 \mu \mathrm{s})$. Based on the energy detectors output over CCA time, the channel is considered as idle or busy. For an idle channel, the LTE-U device starts transmitting immediately, whereas, for a busy channel, more CCAs are performed based on the type of LBT category as follows [18].

- In Category 1, no LBT is performed (similar to CSAT discussed in the next subsection).

- The Category 2 consists of LBT without random back-off.

- An LBT with a random back-off with fixed size of contention window is performed in Category 3.

- $\quad$ The Category 4 consists of LBT with random back-off with variable size of contention window.

The basic LAA-LBT based channel access mechanism is depicted in Figure 2 for the last three categories. The defer period in Figure 2 is the minimum time a device must wait after the channel becomes idle before its transmission [3]. In Figure 2, the four key resource allocation parameters that govern the performance of the LAA-LBT based channel access mechanisms are:

1. the channel access time,

2. the transmit power,

3. the backoff window,

4. the defer period and extended clear channel assessment (ECCA).

A MAC protocol design that to select LAA-LBT's channel access time (also termed as transmission time) such that the LAA-LBT's throughput is maximized while maintaining fairness to existing Wi-Fi devices has been proposed in [19]. Given existing Wi-Fi networks, the feasible region of LAA-LBT's transmission time has also been determined. The impact of ECCA has been neglected in the computation of optimal channel access time in [19]. 


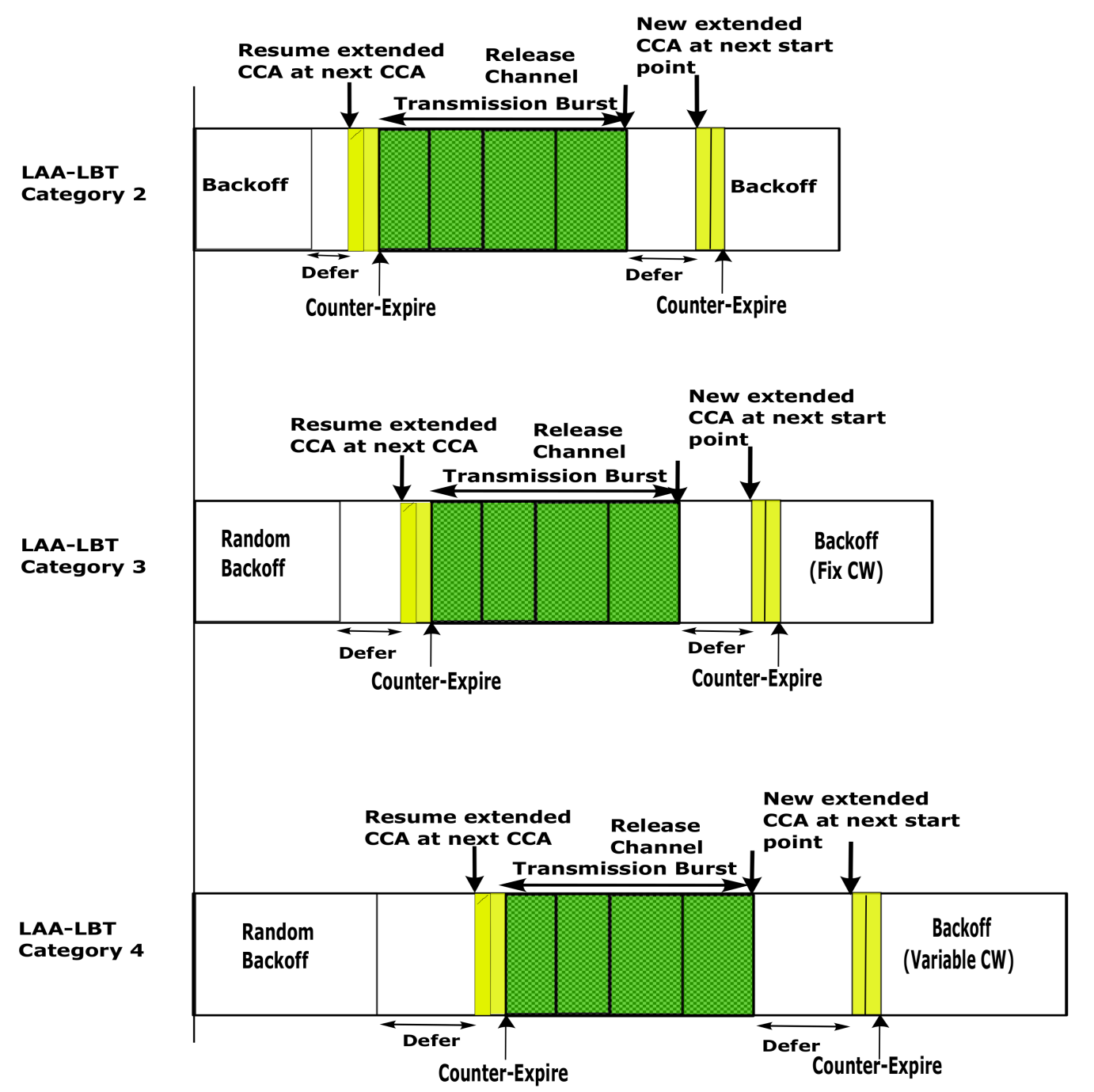

Figure 2. Various LAA-LBT categories for channel access mechanisms of LTE when operating in the unlicensed band.

Joint optimization of the LAA-LBT's channel access time and its transmit power has been considered in [20-22]. The optimal traffic balancing between licensed and unlicensed band for LAA-LBT based SCs has been considered in [20]. The traffic balancing for LAA-LBT and Wi-Fi has been modeled as a coalition game in [23]. Min-max and Shapley fairness has been used as objectives to improve resource utilization with almost blank sub-frames and user offloading for LAA-LBT. A joint subcarrier level power allocation along with channel access time allocation has been formulated as an optimization problem in [21]. Both $[20,21]$ consider a waterfilling algorithm based power allocation.

The tuning of the minimum contention window can have a significant impact on the performance of the overall system. The resource allocation problem for selection of minimum contention window without affecting the aggregate throughput of the WiFi users has been framed in [24]. A Genetic Algorithm based solution has been proposed in [24] to search for the optimal contention window size size of every LAA-LBT SC. Furthermore, a multi-agent reinforcement learning (Q-learning) based contention window adjustment for LAA-LBT has also been proposed in [24] to achieve the dual goals of maximizing the LAA-LBT utility and ensuring the Wi-Fi network's throughput. In [25], a Markov chain-based approach has been used to obtain the optimal contention window size of LTE-U SCs, which maximizes the total throughput of Wi-Fi and LTE-U networks. The carrier selection, discontinuous transmission and transmit power control for LAA-LBT have been considered in [26] with an enhanced Q-learning technique for carrier sensing. In [27], the optimal sizes of the contention 
window have been derived, and a novel resource scheduling approach employing linear programming has been proposed to maximize the spectrum efficiency.

A joint optimization problem considering transmit power, channel access time, and contention window size of the LAA-LBT based SC users that guarantees them a minimum data rate while minimizing the interference to the nearby Wi-Fi users have been presented in [28]. Channel access algorithms utilizing the solution of the joint optimization problem have been proposed in [28]. Furthermore, an algorithm for admission control of LAA-LBT SC users based on the available unlicensed bandwidth from Wi-Fi networks is also discussed Alternatively, a Markov chain based approach has been used to derive the optimal contention window size for LAA based systems in [29]. Furthermore, this optimal contention window size has been used to perform user scheduling with the objective of minimizing the waiting time of the packets in the queue. An Interior Point Algorithm based solution has also been proposed in [27] for user scheduling. In [29], the LTE-U interference has been modeled using a hidden Markov process. The problem of jointly adapting Wi-Fi rates/modes using a framework of partially observable Markov decision process has been solved in [23]. Furthermore, a detection approach based on the sliding window correlation is analyzed for the transmit and sense mode. In [30], resource allocation of dynamically configurable channel occupancy duration and flexible allocation of subcarriers has been considered for an LTE-U system.

In some geographical regions like India, USA, and China, the LAA-LBT is not mandatory [3]. For such regions, the following channel access mechanism has been proposed for the LTE's operation in the unlicensed band.

\subsection{Carrier Sensing Adaptive Transmission (Csat)}

In CSAT, the LTE-U based SC senses the channel for a longer duration compared to LBT and CSMA (around 10 to $200 \mathrm{~ms}$ ) [5]. Then, based on the sensed activity on the channel, the CSAT algorithm selects the proportion of time to be used for LTE as shown in Figure 3. Thus, for a proportion of the gating cycle, the CSAT based LTE-U SC actively utilizes the unlicensed spectrum, while, for the rest of the cycle, the SC switches off the transmission. Note that the CSAT has longer latency then CSMA or LBT, an impact that is mitigated by avoiding channels that Wi-Fi APs use for discovery signals and QoS traffic (i.e., primary channels) [5]. Thus, CSAT can also ensure fair channel sharing between LTE-U and neighboring Wi-Fi devices by suitably selecting the LTE-U ON period as depicted in Figure 3, utilizing optimal transmit power, and balancing the load between the licensed and the unlicensed LTE users.

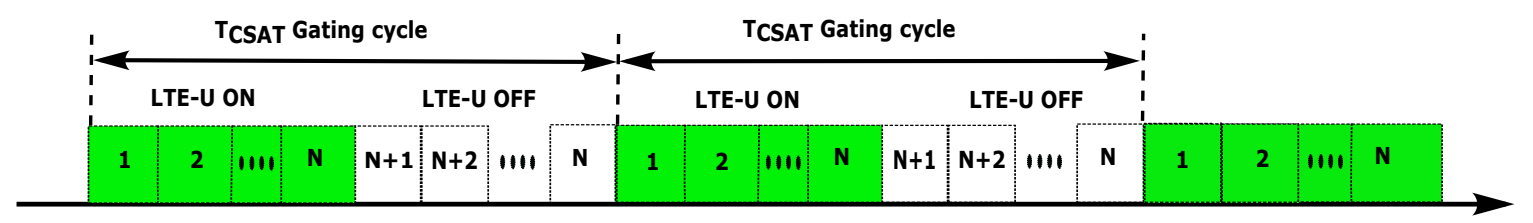

Figure 3. CSAT based channel access for LTE operation in the unlicensed band.

In [31], a proportional fair rate allocation scheme has been considered for a mixed Wi-Fi and LTE-U network. Optimal LTE-U ON time has been derived using the famous Karush-Kuhn-Tucker (KKT) conditions [32] on the rate allocation problem. Similarly, a proportional fair rate allocation for CSAT and LBT has been framed as a convex optimization and solved using the KKT conditions for optimal LTE-U ON time. Joint resource allocation for LTE-U based SC's in the licensed and unlicensed bands has been considered in [33]. A weighted Tchebycheff method based algorithm has also been presented that can find the complete set of Pareto optimal solutions for the energy efficient resource allocation in [33]. In [34], a fair time-sharing model based on the ruin theory has been proposed to perform efficient duty-cycle allocation in LTE-U. 
In [35], a multi-objective joint optimization for load balancing between Wi-Fi and LTE-U has been framed. The optimal solution to the multi-objective optimization includes both the number of users to be offloaded as well as the optimal LTE-U ON time. Even the scenario of multiple Wi-Fi APs has been considered in [35]. Both throughput and fairness for the LTE-U system have been maximized by a multi-objective optimization problem in [36]. In [37], a multi-armed bandit approach for dynamic duty cycle selection has been considered along with downlink power control for higher energy efficiency and interference reduction. In [38], a minority game inspired distributed user association mechanism has been proposed for LTE-U SCs that aims to achieve load balancing under different resource contention schemes between the LTE-U and Wi-Fi networks. Joint optimization of transmit power, available bandwidth, and CSAT channel access time has been considered in [39]. A max-min based objective function has been used to ensure fairness in the joint optimization across both Wi-Fi and LTE networks' throughput. Utilization of the Wi-Fi point coordination function protocol by LTE-U to facilitate fair coexistence with Wi-Fi has been considered in [40]. A network utility maximization problem based on the Nash bargaining solution has been considered in [40], along with joint optimization of user association and resource allocation to improve system throughput and user fairness. A closed-form expression for the optimal contention period of Wi-Fi under a given user association has also been derived in [40]. In [41], a hybrid approach using both CSAT and LAA-LBT has been considered. Joint spectrum and transmission power allocation along with dynamic adaptation of the back-off window size and the duty-cycle time fraction based on the Wi-Fi traffic and available licensed spectrum resource has been considered in [41]. Similarly, analytical derivation of dynamic switching between CSAT and LBT for LTE-U has been considered in [42]. Both CSAT and LAA-LBT as channel access mechanisms can ensure channel sharing between existing devices. However, the $5 \mathrm{GHz}$ band has multiple channels available. Hence, the channel selection for the LTE's operation in the unlicensed band discussed next is critical to avoid interference and ensure fair co-existence.

\section{Channel Selection for LTE Operation in the Unlicensed Band}

The objective of channel selection is to choose one or more $20 \mathrm{MHz}$ channels for LTE operation in the unlicensed band that would cause least interference to the existing devices operating in these channels [43]. The channel selection for LTE operation in the unlicensed band is performed periodically over a time scale larger than the channel access mechanisms discussed in the previous section. Varying numbers of neighboring devices and their corresponding traffic loads play an important role in designing appropriate channel selection schemes. A comprehensive set of scenarios for LTE's channel selection in the unlicensed band is as follows [44]:

- Fully distributed scenario with each LTE based SC independently selecting the channel in the unlicensed band.

- Scenario with intra-operator coordination, such that all SC's of an operator share information and jointly perform channel selection for the LTE's operation in the unlicensed band.

- Inter-operator coordination scenario, where all operators in the vicinity of each other share LTE's channel selection information for the unlicensed band.

- Inter-RAT coordination scenario in which multiple LTE operators share channel selection information even with nearby Wi-Fi networks.

Given the unlicensed channel selection by the LTE operating in the unlicensed band, the selected channel can be used in one of the following three modes [45]:

- Supplemental Downlink: The unlicensed channel is used to carry only data traffic in downlink. The uplink and control channels remain on the licensed spectrum.

- Carrier Aggregation: The uplink and downlink data can be transmitted over both unlicensed and licensed channel. However, the control channel remains in the licensed spectrum. 
- Standalone: Data and control information on the downlink and uplink is sent completely on the unlicensed spectrum.

The different modes of operation for LTE in the unlicensed band are illustrated in Figure 4.

The fully distributed scenario of channel selection for LTE-U with the utilization of the unlicensed band in the supplemental downlink mode has been explored in [44]. A distributed Q-learning based mechanism has been proposed in [44] in which each LTE-U based SC measures and stores an expected reward that can be achieved by using any channel based on its past experience. These reward values are used for efficient channel selection that ensures fair coexistence with Wi-Fi. A Q-learning based channel selection scheme for the fully distributed scenario with supplemental downlink mode has been considered in [46] for non-stationary users. The supplemental downlink mode with the fully distributed scenario has also been modeled as a non-cooperative repeated game in [47]. An iterative trial and error learning based approach for selection of best action has been presented in [47] that drives each SC towards a Nash equilibrium of channel selection.

A cognitive spectrum sharing scheme for carrier aggregation mode in the intra-operator coordination scenario has been proposed in [48]. The spectrum sharing for LTE-U in [48] has been framed with utility maximization as the objective to ensure proportional fairness between the SCs. The resulting formulation turns out to be a mixed-integer nonlinear optimization problem (MINLP) because of the binary nature of channel assignment. Traditionally, the MINLPs are difficult to solve optimally in polynomial time. Hence, a solution algorithm based on a combination of a branch and bound and successive convex relaxation techniques for channel selection has been presented in [48].

A spatial adaptive play based channel selection method has been presented in [49] for the distributed scenario with carrier aggregation mode. The distributed algorithm in [49] tries to achieve the optimal action profile in which each LTE-U SC adapts its channel selection based on neighboring LTE-U SC usage, and this channel selection information is shared in the group. The joint user association, channel selection, and load balancing for LTE-U has been formulated as a non-cooperative game in [50] for the fully distributed scenario with the carrier aggregation mode. Furthermore, a distributed machine learning algorithm based on the echo state networks has been proposed in [50] for channel selection. In [50], it has been shown that the proposed algorithm converges to a mixed strategy Nash equilibrium and performs better than the existing Q-learning based schemes for distributed channel selection.

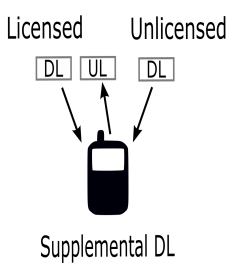

(A)

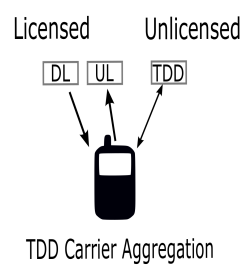

(B)

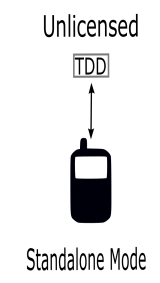

(C)

Figure 4. Different modes of operation for the LTE in the unlicensed band.

Inter-RAT coordination for spectrum sharing between multiple operators using both LTE-U and Wi-Fi have been modeled as a repeated game in [51]. A geographical clustering-based approach for the LTE-U SCs and the Wi-Fi APs has been used for coordination. The coordinating SCs use a decision tree learning based channel selection scheme in [51]. Furthermore, an incentive mechanism has been proposed to motivate operators to willing to share spectrum resources. In [52], a deep learning approach has been proposed for modeling the resource allocation problem of LAA-LBT SCs with dynamic channel selection, carrier aggregation, and fractional spectrum access while guaranteeing fairness with existing Wi-Fi networks. 
Note that channel selection should not be confused with dynamic frequency selection (DFS). The DFS is a regulatory requirement to select a channel on a relatively slow time scale in comparison to channel selection to avoid co-channel interference to existing systems like RADAR [43]. A key issue in LTE in unlicensed band deployment with various channel access mechanisms and channel selection schemes are the fair co-existence of LTE-U devices with other devices in the unlicensed bands. Thus, in the next section, we study the impact of LTE's operation in the unlicensed band on the fairness of the system.

\section{Fairness}

For fair co-existence with Wi-Fi devices, 3GPP mandates that LTE's operation in the unlicensed band must not impact Wi-Fi services any more than an additional Wi-Fi network in the same band [18]. In this regard, fair coexistence of CSAT based LTE-U with Wi-Fi has been studied in $[45,53,54]$. A detailed field trial of CSAT's fair coexistence with Wi-Fi from a normalized throughput perspective for a varying number of users and SC's has been considered in [53]. Both intra-operator and inter-operator scenarios of LTE's operation in unlicensed band with sparse and dense multi-cell deployments show that LTE's operation in unlicensed band outperforms Wi-Fi for all measurement distances considered in [53]. From a delay based analysis, it has been shown in [45], that more work needs to be done to achieve fair coexistence between Wi-Fi and CSAT based LTE-U. In [54], a novel optimal traffic balancing scheme has been proposed for multiple unlicensed bands to achieve fairness between LTE-U and Wi-Fi through optimum power and time allocation in LTE-U.

For a large number of devices, the centralized optimization framework proposed in [39] results in fair sharing between Wi-Fi and LTE's operation in unlicensed band by exploiting power control and time division channel access diversity. Using a max-min fairness based approach, the results in [39] indicate that uncoordinated time based operation of Wi-Fi and LTE's operation in unlicensed band results in severe performance degradation. A central coordinator based approach for fair coexistence between LTE-U and Wi-Fi has been analyzed in [35] for the three scenarios where the central coordinator has no information about Wi-Fi users, has information about the distance between Wi-Fi and LTE-U SC, and has the channel state information. A Nash bargaining solution is used for a fair and joint user association plus resource allocation in [35]. To achieve the various possible performance trade-offs between LTE-U and Wi-Fi, a joint licensed and unlicensed spectrum allocation is considered in [55]. In [31], a joint proportional fair scheduling scheme for coexistence of Wi-Fi and LTE's operation in the unlicensed band has been proposed.

Several works like [49,56-58] analyze CSAT-based channel access. However, they do not consider the impact of a varying number of users, SCs, or multi-operator scenarios on fairness of the system. In [48], the dynamic channel selection, carrier aggregation, and fractional spectrum access for LTE-U networks has been considered, while guaranteeing fair spectrum access for Wi-Fi based on a cross-technology fairness criterion. The per-user average throughput for the LTE users has been maximized in [59], while bounding the minimum average throughput and maximum delay in each Wi-Fi network.

For LAA-LBT, the impact of LTE-U on Wi-Fi for multiple cells and multi-operator scenario has been studied in [60]. Given that Wi-Fi's AIFS time $(34 \mu \mathrm{s})$ is longer than LAA-LBT based LTE-U's proposed CCA time $(20 \mu \mathrm{s})$, there exists scenarios in which LTE-U will sense the channel idle and transmit before any Wi-Fi device. To mitigate these scenarios, a fair LAA-LBT mechanism has been proposed in [6] that maximizes the LTE-U's airtime while not degrading the Wi-Fi's throughput. The gain for LTE's operation in an unlicensed band in [6] increases as the number of competing Wi-Fi stations grow. A novel and fair LAA-LBT algorithm to ensure high throughput of both LTE-U and Wi-Fi has been proposed in [61]. Similarly, an adaptive LAA-LBT has been proposed in [62] that scales fairly with an increasing number of users. In [63], the basic LBT mechanism is used to guarantee fairness with Wi-Fi along with a coalition strategy between LTE-U SCs to decrease the high collision probability at a heavy traffic load. A cooperative game theoretic solution is proposed in which SCs in 
a coalition contend for accessing the channel as single entity. This effectively reduces the number of stations contending for accessing the channel and the collision probability.

A stochastic geometry-based approach for performance analysis of LTE-U and Wi-Fi has been presented in $[64,65]$. The impact of number of users and SCs on the fairness of the system is evaluated in $[64,65]$, respectively. A stochastic geometry-based framework has been considered in [66] to analyze the throughput and fairness issues in Wi-Fi and LTE-U's coexistence.

A discrete Markov chain-based analysis of the LAA-LBT based system is considered in $[61,67]$ that shows fair co-existence with an increasing number of users and SCs, respectively. In [68-71], the fairness of LAA-LBT based LTE-U with respect to existing Wi-Fi users has been considered. However, the impact of increasing number of users, SCs, and operators needs to be further studied.

The number of studies that analyze the fairness of LAA-LBT based channel access mechanism are larger than a similar number of studies for CSAT based channel access. However, a comprehensive study that analyzes LAA-LBT based LTE-U's fairness towards Wi-Fi with varying number of users, $\mathrm{SCs}$, and operators is required. A comprehensive summary of the various studies on the fairness of CSAT and LAA-LBT based channel access mechanisms along with various channel selection schemes is presented in Table 1. Next, we explore the existing studies from the perspective of coverage, capacity, and coordination in LTE's operation in the unlicensed band.

Dynamic uplink radio access selection scheme between scheduling and random access for LAA uplink transmission has been proposed in [72]. In order to exploit optimum performance, an optimization game of the uplink radio access selection has been formulated. The optimum solution of the game is proved to exist by potential game theory. The comparison of fairness between LBT and CSAT has been performed in [73]. The fundamental trade-off of co-channel interference (CI) in a licensed spectrum and collision probability on the unlicensed spectrum has been analyzed by developing a power allocation rule with double water-filling lines, and the complete set of Pareto-optimal solution has been achieved by the weighted Tchebycheff method in [22]. In [74], the LTE-U sum-rate maximization has been addressed using a cooperative Nash bargaining game for fair coexistence and a one-sided matching game for a resources allocation problem in the LTE-U system. The two games are repeated until convergence is achieved in [74]. 
Table 1. A summary of fair co-existence analysis of LTE with WiFi on the unlicensed band.

\begin{tabular}{|c|c|c|c|c|c|}
\hline & \multirow{6}{*}{ CSAT Based } & Scale with users & Scale with APs/BSs & Scale with Operators & References \\
\hline \multirow{10}{*}{ Channel Access Mechanisms } & & $\checkmark$ & $\checkmark$ & $\checkmark$ & {$[35,53]$} \\
\hline & & $\checkmark$ & $X$ & $X$ & {$[19,31,54,55]$} \\
\hline & & $x$ & $\checkmark$ & $x$ & [48] \\
\hline & & $\checkmark$ & $\checkmark$ & $X$ & {$[39,50,66]$} \\
\hline & & & Not specified & & {$[49,58]$} \\
\hline & \multirow{5}{*}{ LAA-LBT Based } & $\checkmark$ & $\checkmark$ & $X$ & {$[27,28]$} \\
\hline & & $X$ & $\checkmark$ & $\checkmark$ & \\
\hline & & $\checkmark$ & $\mathrm{X}$ & $X$ & {$[6,21,42,61,62,64]$} \\
\hline & & $X$ & $\checkmark$ & $x$ & {$[23,25,63,65,67,73]$} \\
\hline & & & Not specified & & {$[20,26,33,41,68-71]$} \\
\hline \multirow{6}{*}{ Channel Selection Schemes } & \multicolumn{4}{|c|}{ Type of Scheme } & References \\
\hline & \multicolumn{4}{|c|}{ Coordinated approach for joint channel selection } & {$[57,75]$} \\
\hline & \multicolumn{4}{|c|}{ Spatial adaptive play based channel selection method } & [49] \\
\hline & \multicolumn{4}{|c|}{ Mode selection } & [29] \\
\hline & \multicolumn{4}{|c|}{ Repeated game and learning based channel selection } & [47] \\
\hline & \multicolumn{4}{|c|}{ Q-learning based channel selection } & {$[44,46]$} \\
\hline
\end{tabular}




\section{Coverage, Capacity, and Coordination in LTE-U}

The SINR coverage of an LTE-U operator is defined as the probability that a randomly chosen UE can achieve an SINR through LTE-U SC higher than a pre-determined SINR threshold as given by [65]. The analytical results in [65] are presented for a single carrier, random carrier selection, and adaptive carrier selection schemes. In [76], SINR and rate coverage probabilities have been analytically derived and numerically evaluated for a stochastic geometry based framework. It has been shown in [76] that WLAN and LTE-U can jointly achieve better rate coverage probability than typical WLAN networks through lower channel access priority along with a more sensitive sensing threshold for LTE-U. In [77], network capacity has been optimized for LTE-U and Wi-Fi coexistence with an optimal spectrum splitting ratio. Furthermore, the coverage probabilities and average achievable rates for each radio access technologies have been derived based on the user association in the networks.

In [53], coverage and capacity performance has been evaluated through field trials for LTE-U and WLAN for single and multi-operator scenarios. A similar field trial has been considered in [78] for ultra-dense networks. Four different scenarios for the LTE-U SCs and corresponding transmission powers have been considered in [53] as follows:

- For coverage test: $20 \mathrm{dBm}$,

- For capacity test: $16 \mathrm{dBm}$,

- For single-operator test: $8 \mathrm{dBm}$,

- For multi-operator test: $16 \mathrm{dBm}$.

The authors in [53] show that direct introduction of LTE-U is not recommended and interference avoidance technology is essential to maintain coverage and capacity in LTE-U. Furthermore, it has been shown in [4] that existing water-filling algorithms are not suitable for LTE-U, due to computation load and possible iteration divergence. Hence, to reduce complexity and achieve capacity, a successive cap-limited water-filling algorithm has been proposed in [4] that maximizes the weighted capacity within the power and interference constraints. In [79], a weighted Voronoi tessellation-based approach has been utilized to maximize network capacity over an optimal SC operation scheme in LTE-U.

Typically, the WLANs are distributed networks based on the DCF. However, the LTE-U SC based cellular networks can be centralized or distributed. The hardware based results in [39] indicate that both WLAN and LTE networks suffer due to interference from each other. Hence, inter-network coordination between WLAN and LTE-U through a centralized radio resource management entity has been proposed in [39] for improved system performance. A novel approach of applying coordinated multipoint (COMP) based LTE-U has been considered in [80]. The simulation results in [58] show that interference coordination can significantly improve the ergodic throughput of LTE-U without compromising on WLAN's throughput. Coordinated and distributed channel selection for collocated WLAN and LTE-U cells has been analyzed in [57] for a single operator scenario. However, a similar study for multi-operator scenario is required. A detailed study of intercell interference coordination (ICIC) for LTE-U and WLAN has been performed in [7] with the following four scenarios.

- Scenario 1 (Worst Coordination) Non Co-Located SCs with fully time aligned CSAT gating cycle.

- Scenario 2 (Worst Coordination) Co-Located SCs with fully time aligned overlapping CSAT gating cycle.

- Scenario 3 (Best Coordination) Co-Located SCs using proposed ICIC in [7] to get orthogonal non-overlapping CSAT gating cycle.

- Scenario 4 (Random No Coordination) Co-Located SCs using randomly partially aligned overlapping CSAT gating cycle.

The scheme proposed in [7] results in improvement of the overall LTE-U system performance. In [35], a central coordinator based approach for fair coexistence between LTE-U and WLAN has been analyzed based on the availability of distance and channel state information of the users at the central 
coordinator. A novel cloud based centralized resource allocation for LAA-LBT has been proposed in [81]. The centralized scheme in [81] achieves the optimum resource utilization in the time and spatial domain along with optimized energy efficiency.

An important issue from a coordination perspective in LTE-U is to decide between a frame based equipment (FBE) or load based equipment (LBE). In an FBE, the LTE-U frames align with the existing LTE frame structure. Alternatively, an LBE can contend for the channel as soon as it has data to transmit. From LTE-U perspective, it has been discussed in [69] that an FBE can result in excessive delays for high system load. Intra-operator and inter-operator synchronization in LTE-U with FBE can result in multiple LAA cells observing the same resources as free causing frequent collisions [69]. Thus, an LBE is more suitable for LTE-U. A distributed scheme with a mix of LBT and CSAT has been considered in [82] for channel reservation during the spectrum sensing period and LTE-U transmissions. The LTE-U transmission in [82] is followed by the reserved duration for WLAN transmissions. It has been shown in [82] that a reasonable level of WLAN protection can be achieved by adapting the LTE-U parameters. In [49], a local interaction game based approach has been used for distributed channel selection in LTE-U. Furthermore, an optimal spatial adaptive play (SAP) based LTE-U channel selection algorithm is proposed in [49]. Distributed channel access mechanisms have been evaluated in $[6,67,68]$. Distributed channel selection has been examined in $[44,46,60]$. Next, we analyze the existing LTE-U studies from the perspective of the environment, traffic type, and scenarios considered.

\section{LTE-U's Evaluation in Diverse Traffic Type, Environments, and Scenarios}

The LTE-U performance for a full buffer or saturated mode has been analyzed in $[39,53]$ for downlink traffic with time division duplexing (TDD). A similar analysis for LTE-U with full buffer downlink and frequency division duplexing (FDD) has been performed in [31]. However, a joint analysis for LTE-U comparing downlink traffic with a full buffer for both FDD and TDD is not available. A significant number of works as shown in Table 2 consider the saturated traffic conditions but do not clearly demarcate the TDD or FDD as the duplexing mode. Furthermore, in the full buffer scenario, no analysis is available for LTE-U with uplink traffic.

Table 2. A summary of traffic models studied for LTE-U.

\begin{tabular}{|c|c|c|c|}
\hline Buffer & Uplink Downlink & Comment & References \\
\hline \multirow{4}{*}{ Saturated } & Uplink & NA & {$[50]$} \\
\hline & \multirow{3}{*}{ Downlink } & TDD & {$[39,53]$} \\
\hline & & FDD & [31] \\
\hline & & Not specified & {$[6-8,20,21,29,35,44,50,57,60,66,67,76,82,83]$} \\
\hline \multirow{5}{*}{ Non-saturated } & Uplink & TDM & {$[81]$} \\
\hline & \multirow{3}{*}{ Downlink } & Voice traffic & {$[14,27,43,69]$} \\
\hline & & FTP traffic & {$[14,69,84]$} \\
\hline & & Not specified & {$[6,40,48,58,68,70,78,85]$} \\
\hline & \multicolumn{2}{|c|}{ Not specified } & {$[54,71,86]$} \\
\hline
\end{tabular}

In contrast with the full buffer mode, unsaturated traffic conditions for uplink traffic with TDM have been studied in [81] for an FBE. The impact of co-existence of WLAN and LTE-U for FTP and VoIP Packet data at WLAN and downlink FTP data at LTE-U has been analyzed in [69]. In [43], both FTP and VoIP data over the LTE-U downlink have been analyzed in the presence of both uplink and downlink packet transfer in the WLAN. FTP data based downlink data traffic for LTE and WLAN have been considered in [84]. Although [54,71,86] consider models that are inspired by unsaturated traffic conditions, more detailed study of LTE-U with various channel access mechanisms and channel selection schemes in the presence of diverse traffic type is required. 
The performance of LTE-U based SCs in the presence of WLAN has been evaluated for both indoor and outdoor environments in $[43,60,64,70,71]$. A thorough evaluation specifically for indoor deployment of LTE-U based SCs has been considered in $[3,39,44,46,53,68,69]$. However, in depth studies for outdoor deployment of LTE-U SCs coexisting with WLAN has been analyzed in $[7,57,83,84]$. The work in [80] in particular presents results consider realistic deployments of LTE-U based SCs.

Most of the simulation based studies for LTE-U like $[3,4,7,60]$ have considered MATLAB as the simulation environment. However, packet level simulation with the LTE-LENA stack and NS have been considered in [87]. The WaltSystem and WinProp based simulation results have been shown in $[57,86]$, respectively, while [80] presents simulation results for both MATLAB and WinProp based simulations. Detailed hardware based implementation of LTE-U using USRPs and the ORBIT testbed has been considered in [39]. Similarly, NTT DOCOMO and Huawei's field trial results using proposed LTE-U hardware has been shown in [53]. Details of various simulation, hardware, traffic environments, and scenarios considered are summarized in Tables 2 and 3. Next, we consider the important issues of latency and handover analysis for LTE-U based SCs.

Table 3. A summary of evaluation environments considered for LTE-U performance analysis.

\begin{tabular}{|c|c|c|c|c|}
\hline & Indoor/Outdoor & Coordinated/Distributed & Comment & References \\
\hline \multirow{6}{*}{$\begin{array}{c}\text { Simulation } \\
\text { based }\end{array}$} & \multirow{2}{*}{ Indoor } & \multirow{2}{*}{ Distributed } & WaltSystem & [86] \\
\hline & & & MATLAB & {$[3,60]$} \\
\hline & \multirow{4}{*}{ Outdoor } & Coordinated & MATLAB & {$[7,80]$} \\
\hline & & \multirow{2}{*}{ Distributed } & MATLAB & {$[4,60,80]$} \\
\hline & & & NS & [87] \\
\hline & & Both & WinProp & {$[57,80]$} \\
\hline \multirow{3}{*}{$\begin{array}{c}\text { Hardware } \\
\text { implementations }\end{array}$} & \multicolumn{3}{|c|}{ Experimental evaluations using USRP-based SDR on the ORBIT testbed } & [39] \\
\hline & \multicolumn{3}{|c|}{ NTT DOCOMO and Huawei's field trials for LTE-U } & [53] \\
\hline & \multicolumn{3}{|c|}{ UE of HUAWEI used as U-LTE terminal } & [78] \\
\hline
\end{tabular}

\section{Latency and Handover Analysis for LTE-U}

The performance gains in terms of throughput and coverage from the operation of LTE-U SC's in the unlicensed spectrum have been discussed in the previous sections. However, network latency is another key network performance indicator that must be studied for LTE-U. In this direction, the average number of collisions and latency analysis for WLAN and CSAT based LTE-U has been presented in [88]. It has been shown in [88] that an increase in the CSAT duty cycle results in increased WLAN latency. Buffer occupancy and delay CDF's under low, medium, and high load conditions for LAA-LBT based LTE-U have been studied in [68]. The delay and throughput performance of WLAN in the presence of dynamic contention window adjusting LAA-LBT have been analyzed in [3,71]. It has been shown that dynamic contention window adjustment in LAA-LBT results in fair coexistence with WLAN. In [89], the ratio of latency to total channel occupancy time has been studied. It has been shown that, from a delay perspective, FBE performs better than LBE in LTE-U. Furthermore, it has been shown that appropriately tuning the duty cycle in CSAT can result in better delay performance.

Given LTE-U based SCs, another key aspect of the network is the frequent handover in the presence of mobile users. A modified version of the LENA LTE module in the ns-3 simulator is used in [87] to evaluate the TCP's performance for LTE-U handover scenarios. The results in [87] indicate that a new TCP mechanism is needed for coexistence between LTE-U and LTE. In [90], the effect of handover trigger in LTE-U due to the unavailability of unlicensed band in the SC has been considered. Furthermore, a new handover scheme has been proposed and analyzed for LAA 
networks. Hence, more research is required in handover analysis for LTE-U. Next, we discuss the operation in the unlicensed spectrum of the upcoming $5 \mathrm{G}$ new radio.

\section{5G New Radio}

For the upcoming 5G new radio (NR), 3GPP has approved the operation in the existing $5 \mathrm{GHz}$ unlicensed band and the new 'greenfield' $6 \mathrm{GHz}$ unlicensed band. Five key scenarios for 5G NR in the unlicensed band (also termed as NR-U) are [91-94]:

- Carrier Aggregation within gNodeB,

- Dual connectivity across eNodeBs,

- LTE anchor in licensed spectrum,

- 5G NR anchor in licensed spectrum,

- Uplink only in licensed spectrum,

- Downlink only in unlicensed spectrum with the stand-alone operation.

The licensed-assisted access NR-U (LAA NR-U) scenario aggregates an unlicensed spectrum with licensed spectrum, which enables operators to boost the network performance speed and capacity. It has been demonstrated in [95-100] that 5G NR boosts the network speed and capacity. Furthermore, key issues like handover, antenna designing, and spectrum management along with possible solutions for 5G NR have been discussed in [95-100]. Note that the LAA NR-U supports both NR and LTE in a licensed spectrum combined with NR-U in unlicensed spectrum. The LAA NR-U can be deployed by a macro-cell using licensed spectrum, and a local small-cell using an unlicensed spectrum or by using carrier aggregation in a small-cell (supporting both licensed and unlicensed spectrum). The 5G NR operating in the unlicensed spectrum will broaden the 5G vision to expand and transform industries. These industries are dedicated to a specific application such as industrial Internet-of-Things (IoT) and mobile broadband for enterprises.

Network slicing is one of the key enabling technologies for 5G NR due to its ability to customize and 'slice' a common resource to support diverse services and verticals [101]. An inter-operator network slicing framework for multiple mobile network operators (MNOs) has been considered in [101] in both licensed and unlicensed bands. Cellular vehicle-to-everything (V2X) communications is another key technology considered in 5G NR [102]. In [102], the coexistence problem of cellular vehicle-to-everything (V2X) users and vehicular ad hoc network (VANET) users over the unlicensed spectrum have been considered. However, detailed studies for resource allocation of LAA NR-U are needed. The ability to operate 5G NR stand-alone in an unlicensed spectrum is the next important scenario. A possible candidate technology for 5G NR's stand-alone cellular operation in an unlicensed spectrum is called MulteFire and discussed in the following section. In [37], an experimental testbed for indoor co-existence of LTE-U and Wi-Fi has been considered. Coverage, capacity, clear channel assessment energy detection threshold for LTE-U along with supplemental downlink and cell sleeping have been investigated to verify the fair coexistence between LAA and Wi-Fi in the unlicensed spectrum. A virtual coalition formation game approach has been used in [103] to solve the unlicensed band selection problem in 5G.

\section{Multefire}

MulteFire is an LTE-based technology built on elements of the 3GPP Release 13 and 3GPP Release 14 specifications for LAA and Enhanced LAA (eLAA). MulteFire operates in standalone mode within the unlicensed spectrum. The main advantage of MulteFire is that it allows anyone to deploy and operate their private network, which can revolutionize the industrial Internet-of-things (IoT). In MultiFire 1.1 release [104], it has been mentioned that it is designed to coexist efficiently with other technologies like Wi-Fi, Licensed Assisted Access (LAA), and Listen-Before-Talk (LBT). In [105,106], an overview of coexistence has been discussed for wireless technology, commercial medical devices, and performance evaluation of a standalone LTE network in an unlicensed band that has been tested 
in [107]. The Markov-Chain model for the LBT mechanism of LTE-LAA according to the 3GPP release 13 and 14 that has been proposed in [108] applies to MulteFire. However, Multefire supports new bands such as $1.9 \mathrm{GHz}$ for Japan and other regional band ranges in 800-900 MHz. Moreover, MulteFire has introduced two key features such as an Autonomous UE Mobility (AUM) and Self-Organizing Networks (SON), in order to complement the typical eNB controlled handover procedure and the network optimization of stand-alone networks operating in unlicensed spectrum and networks deployed with the Neutral Host Network (NHN) architecture, respectively. Additionally, MultiFire focuses on IoT and Expanded IoT services to fulfill their specific requirements for performance, security, latency, and mobility with low power wide area support. In [109-111], a performance comparison of MulteFire with previous technologies has been presented with numerical analysis and system-level simulations. The results have shown that MulteFire can double the capacity and provide more than twice the coverage of Wi-Fi. However, resource allocation for standalone operation of cellular technologies like MulteFire needs more attention from the research community. In [85], a time and frequency division algorithm has been proposed to eliminate the control signaling collisions in standalone LTE-U systems. Next, we discuss the existing implementation/field trial results for cellular operation in unlicensed spectrum.

\section{Open Issues in LTE-U}

A comprehensive study for CSAT based LTE-U exists in the form of [53] in which the fair coexistence of LTE-U has been evaluated with respect to scaling in the number of devices, SCs, and operators. However, a similar comprehensive study for LAA-LBT based LTE-U is needed. From an LAA-LBT perspective, optimal contention window adjustment based on the operating environment needs more research [3]. The optimal channel sense threshold is another key issue in LAA-LBT that requires more attention as discussed in [60,68]. From the CSAT perspective, future research is required to define a collision-free slot assignment for LTE-U in the presence of multiple LTE-U networks. Learning techniques in $[35,83,84]$ can provide distributed collision-free operation. However, further evaluation of these techniques is needed in diverse scenarios for LTE-U [31].

A more thorough investigation of LTE-U's coexistence with technologies besides WLAN (like RADAR as shown in $[2,88]$ ) is required. A comparative study between the different channel access mechanism for LTE-U is also needed, particularly the LBT and CSAT [2]. Furthermore, more field trials with multi-operator and multi-cell LTE-U deployment is required for performance evaluation of the coexistence mechanisms.

From a channel selections perspective, both intra and inter-operator coordination needs to be studied in detail for LTE-U along with the stability analysis of the channel selection schemes [44]. The appropriate selection of licensed and unlicensed bands for the operation of LTE-U users needs to be designed for effective utilization of LTE-U's potential as a technology [54]. The study of channel selection schemes in a multi-cell, multi-operator, and multiple WLAN AP environment is even more important to ensure successful commercial deployment of LTE-U based SC's.

Development of suitable analytical models for various non-saturated traffic models is another important issue that requires more attention in LTE-U [39]. The inherent PHY/MAC differences between LTE-U and WLAN systems must be considered in these models along with inter-cell interference in dense deployments [8]. These models should also consider more practical aspects of LTE-U and WLAN like adaptive modulation and coding, hidden and exposed node problem, etc. [35]. From a practical deployment perspective, specific QoS demands, relay based communication [4], Multiple-Input Multiple-Output (MIMO) systems, and power control [75] are some additional aspects to be studied for LTE-U. A thorough study of both TDD and FDD based LTE-U, with different intra and inter-operator level coordination, is required [61]. Furthermore, the performance of LTE-U for various handover scenarios needs to be evaluated. A novel approach of licensed and unlicensed spectrum 
utilization for D2D communication and IoT has been considered in [112]. However, a more detailed study is needed.

\section{Conclusions}

In this article, we have presented a detailed survey of existing techniques for LTE-U in various scenarios. We have explained the channel access mechanisms and several channel selection schemes. We have studied the performance of these techniques with respect to fairness towards IEEE 802.11 based WLAN, coverage, and capacity. From existing studies, it is apparent that, when tuned properly, the LTE-U not only co-exists fairly with WLAN but can also result in better performance. We have performed a detailed analysis of the impact of traffic type, environment scenarios, and coordination on LTE-U's performance. The latency and handover issues for LTE-U have also been discussed. Through a discussion on open issues in LTE-U, we have presented the possible research directions that need more attention.

Author Contributions: M.M. and A.Y. were involved in planning of the work. M.M., A.K. and A.Y. processed the data, performed the analysis, drafted the manuscript, and M.M. designed the figures. A.K. drafted the Multefire and Open issues in LTE-U section. M.D. and A.K. aided in improvising the manuscript. All authors discussed the results and commented on the manuscript. All authors have read and agreed to the published version of the manuscript.

Funding: This research received no external funding.

Conflicts of Interest: The authors declare no conflict of interest.

\section{References}

1. Peha, J. Approaches to spectrum sharing. IEEE Commun. Mag. 2005, 43, 10-12. [CrossRef]

2. Labib, M.; Reed, J.H.; Martone, A.F.; Zaghloul, A.I. Coexistence between radar and LTE-U systems: Survey on the $5 \mathrm{GHz}$ band. In Proceedings of the United States National Committee of URSI National Radio Science Meeting (USNC-URSI NRSM), Boulder, CO, USA, 4-6 January 2016; pp. 1-2.

3. Baswade, A.M.; Tamma, B.R. Channel sensing based dynamic adjustment of contention window in LAA-LTE networks. In Proceedings of the International Conference on Communication Systems and Networks (COMSNETS), Bangalore, India, 5-10 January 2016; pp. 1-2.

4. Xu, W.; Li, B.; Xu, Y.; Lin, J. Lower-Complexity Power Allocation for LTE-U Systems: A Successive Cap-Limited Waterfilling Method. In Proceedings of the IEEE Vehicular Technology Conference (VTC Spring), Glasgow, UK, 11-14 May 2015; pp. 1-6.

5. LTE in Unlicensed Spectrum Research. Available online: https://www.qualcomm.com/invention/research/ projects/lte-unlicensed (accessed on 15 October 2016).

6. Valls, V.; Garcia-Saavedra, A.; Costa, X.; Leith, D.J. Maximizing LTE Capacity in Unlicensed Bands (LTE-U/LAA) While Fairly Coexisting With 802.11 WLANs. IEEE Commun. Lett. 2016, 20, 1219-1222. [CrossRef]

7. Khawer, M.R.; Tang, J.; Han, F. usICIC-A Proactive Small Cell Interference Mitigation Strategy for Improving Spectral Efficiency of LTE Networks in the Unlicensed Spectrum. IEEE Trans. Wirel. Commun. 2016, 15, 2303-2311. [CrossRef]

8. Zhang, R.; Wang, M.; Cai, L.X.; Zheng, Z.; Shen, X.; Xie, L.L. LTE-unlicensed: The future of spectrum aggregation for cellular networks. IEEE Wirel. Commun. 2015, 22, 150-159. [CrossRef]

9. Al-Dulaimi, A.; Al-Rubaye, S.; Ni, Q.; Sousa, E. 5G Communications Race: Pursuit of More Capacity Triggers LTE in Unlicensed Band. IEEE Veh. Technol. Mag. 2015, 10, 43-51. [CrossRef]

10. The Global Mobile Suppliers Association, LTE in Unlicensed Spectrum: Trials, Deployments and Devices. Available online: https://gsacom.com/paper/lte-unlicensed-spectrum-trials-deploymentsdevices/ (accessed on 15 April 2017).

11. Chen, B.; Chen, J.; Gao, Y.; Zhang, J. Coexistence of LTE-LAA and Wi-Fi on $5 \mathrm{GHz}$ with Corresponding Deployment Scenarios: A Survey. IEEE Commun. Surv. Tutor. 2017, 19, 7-32. [CrossRef]

12. Zhang, H.; Dong, Y.; Cheng, J.; Hossain, M.J.; Leung, V.C.M. Fronthauling for 5G LTE-U Ultra Dense Cloud Small Cell Networks. IEEE Wirel. Commun. 2016, 23, 48-53. [CrossRef] 
13. Falconetti, L.; Karaki, R.; Obregon, E.; Cheng, J.F.; Koorapaty, H.; Mukherjee, A.; Larsson, D. Design and Evaluation of Licensed Assisted Access LTE in Unlicensed Spectrum. IEEE Wirel. Commun. 2016, 23, 24-30. [CrossRef]

14. Kwon, H.J.; Jeon, J.; Bhorkar, A.; Ye, Q.; Harada, H.; Jiang, Y.; Oh, J. Licensed-Assisted Access to Unlicensed Spectrum in LTE Release 13. IEEE Commun. Mag. 2017, 55, 201-207. [CrossRef]

15. Cui, H.; Leung, V.C.M.; Li, S.; Wang, X. LTE in the Unlicensed Band: Overview, Challenges, and Opportunities. IEEE Wirel. Commun. 2017, 24, 99-105. [CrossRef]

16. IEEE. Wireless LAN Medium Access Control (MAC) and Physical Layer (PHY) Specifications. IEEE Standards Association. Standard 802.11. Available online: https://ci.nii.ac.jp/naid/10024984601/ (accessed on 15 June 2012).

17. 3GPP TR 36.899 TSG RAN. Study on Licensed-Assisted Access to Unlicensed Spectrum. Rel.13, v0.1.1. Available online: https://portal.3gpp.org/desktopmodules/Specifications/SpecificationDetails.aspx? specificationId=2579 (accessed on 15 November 2014).

18. RP-150271 Status Report to TSG: Study on Licensed-Assisted Access to Unlicensed Spectrum. 3GPP RAN 67. Available online: https:/ / mentor.ieee.org/802.19/dcn/15/19-15-0042-00-0000-study-on-licensed-assistedaccess-to-unlicensed-spectrum.pdf (accessed on 15 May 2015).

19. Hamidouche, K.; Saad, W.; Debbah, M. A Multi-Game Framework for Harmonized LTE-U and WiFi Coexistence over Unlicensed Bands. IEEE Wirel. Commun. 2016, 23, 62-69. [CrossRef]

20. Liu, F.; Bala, E.; Erkip, E.; Beluri, M.C.; Yang, R. Small-Cell Traffic Balancing Over Licensed and Unlicensed Bands. IEEE Trans. Veh. Technol. 2015, 64, 5850-5865. [CrossRef]

21. He, H.; Shan, H.; Huang, A.; Cai, L.X.; Quek, T. Proportional Fairness-Based Resource Allocation for LTE-U Coexisting with Wi-Fi. IEEE Access 2017, 5, 4720-4731. [CrossRef]

22. Yin, R.; Yu, G.; Maaref, A.; Li, G.Y. A Framework for Co-Channel Interference and Collision Probability Tradeoff in LTE Licensed-Assisted Access Networks. IEEE Trans. Wirel. Commun. 2016, 15, 6078-6090. [CrossRef]

23. Chung, B.C.; Cho, D.H. Mobile Data Offloading with Almost Blank Subframe in LTE-LAA and Wi-Fi Coexisting Networks based on Coalition Game. IEEE Commun. Lett. 2017, 21, 608-611. [CrossRef]

24. Li, Y.; Zhou, T.; Yang, Y.; Hu, H.; Hamalainen, M. Fair Downlink Traffic Management for Hybrid LAA-LTE/Wi-Fi Networks. IEEE Access 2016, 5, 7031-7041. [CrossRef]

25. Song, Y.; Sung, K.W.; Han, Y. Coexistence of Wi-Fi and Cellular With Listen-Before-Talk in Unlicensed Spectrum. IEEE Commun. Lett. 2016, 20, 161-164. [CrossRef]

26. Galanopoulos, A.; Foukalas, F.; Tsiftsis, T.A. Efficient Coexistence of LTE With WiFi in the Licensed and Unlicensed Spectrum Aggregation. IEEE Trans. Cogn. Commun. Netw. 2016, 2, 129-140. [CrossRef]

27. Zhang, Q.; Wang, Q.; Feng, Z.; Yang, T. Design and Performance Analysis of a Fairness-Based License-Assisted Access and Resource Scheduling Scheme. IEEE J. Sel. Areas Commun. 2016, 34, 2968-2980. [CrossRef]

28. Yin, R.; Yu, G.; Maaref, A.; Li, G.Y. LBT-Based Adaptive Channel Access for LTE-U Systems. IEEE Trans. Wirel. Commun. 2016, 15, 6585-6597. [CrossRef]

29. Hirzallah, M.; Afifi, W.; Krunz, M. Full-duplex-based Rate/Mode Adaptation Strategies for Wi-Fi/LTE-U Coexistence: A POMDP Approach. IEEE J. Sel. Areas Commun. 2017, 35, 20-29. [CrossRef]

30. Yuan, J.; Huang, A.; Shan, H. Resource Management of LTE-U Systems for Channel Utilization and User Satisfaction. IEEE Access 2019, 7, 107473-107490. [CrossRef]

31. Cano, C.; Leith, D.J. Coexistence of WiFi and LTE in unlicensed bands: A proportional fair allocation scheme. In Proceedings of the IEEE International Conference on Communication Workshop (ICCW), London, UK, 8-12 June 2015; pp. 2288-2293.

32. Kuhn, H.W.; Tucker, A.W. Nonlinear programming. In Proceedings of the 2nd Berkeley Symposium; University of California Press: Berkeley, CA, USA, 1951; pp. 481-492.

33. Chen, Q.; Yu, G.; Yin, R.; Maaref, A.; Li, G.Y.; Huang, A. Energy Efficiency Optimization in Licensed-Assisted Access. IEEE J. Sel. Areas Commun. 2016, 34, 723-734. [CrossRef]

34. Manzoor, A.; Tran, N.H.; Saad, W.; Kazmi, S.M.A.; Pandey, S.R.; Hong, C.S. Ruin Theory for Dynamic Spectrum Allocation in LTE-U Networks. IEEE Commun. Lett. 2019, 23, 366-369. [CrossRef]

35. Chen, Q.; Yu, G.; Maaref, A.; Li, G.; Huang, A. Rethinking Mobile Data Offloading for LTE in Unlicensed Spectrum. IEEE Trans. Wirel. Commun. 2016, 15, 4987-5000. [CrossRef] 
36. Wu, W.; Yang, Q.; Liu, R.; Kwak, K.S. Protocol Design and Resource Allocation for LTE-U System Utilizing Licensed and Unlicensed Bands. IEEE Access 2019, 7, 67068-67080. [CrossRef]

37. Sriyananda, M.G.S.; Parvez, I.; Güvene, I.; Bennis, M.; Sarwat, A.I. Multi-armed bandit for LTE-U and WiFi coexistence in unlicensed bands. In Proceedings of the 2016 IEEE Wireless Communications and Networking Conference, Doha, Qatar, 3-6 April 2016; pp. 1-6.

38. Wang, Y.; Yuan, J.; Yu, G.; Chen, Q.; Yin, R. Minority Game for Distributed User Association in Unlicensed Heterogenous Networks. IEEE Trans. Wirel. Commun. 2020, 19, 4220-4233. [CrossRef]

39. Sagari, S.; Baysting, S.; Saha, D.; Seskar, I.; Trappe, W.; Raychaudhuri, D. Coordinated dynamic spectrum management of LTE-U and Wi-Fi networks. In Proceedings of the IEEE International Symposium on Dynamic Spectrum Access Networks (DySPAN), Stockholm, Sweden, 29 September-2 October 2015; pp. 209-220.

40. Chen, Q.; Yu, G.; Ding, Z. Optimizing Unlicensed Spectrum Sharing for LTE-U and WiFi Network Coexistence. IEEE J. Sel. Areas Commun. 2016, 34, 2562-2574. [CrossRef]

41. Liu, S.; Yin, R.; Yu, G. Hybrid Adaptive Channel Access for LTE-U Systems. IEEE Trans. Veh. Technol. 2019, 68, 9820-9832. [CrossRef]

42. Lien, S.Y.; Lee, J.; Liang, Y.C. Random Access or Scheduling: Optimum LTE Licensed-Assisted Access to Unlicensed Spectrum. IEEE Commun. Lett. 2016, 20, 590-593. [CrossRef]

43. Mukherjee, A.; Cheng, J.F.; Falahati, S.; Falconetti, L.; Furuskär, A.; Godana, B.; Yang, Y. System architecture and coexistence evaluation of licensed-assisted access LTE with IEEE 802.11. In Proceedings of the IEEE International Conference on Communication Workshop (ICCW), London, UK, 8-12 June 2015; pp. 2350-2355.

44. Sallent, O.; Perez-Romero, J.; Ferrus, R.; Agusti, R. Learning-based coexistence for LTE operation in unlicensed bands. In Proceedings of the IEEE International Conference on Communication Workshop (ICCW), London, UK, 8-12 June 2015; pp. 2307-2313.

45. Babaei, A.; Andreoli-Fang, J.; Hamzeh, B. On the impact of LTE-U on Wi-Fi performance. In Proceedings of the IEEE Annual International Symposium on Personal, Indoor, and Mobile Radio Communication (PIMRC), Washington, DC, USA, 2-5 September 2014; pp. 1621-1625.

46. Perez-Romero, J.; Sallent, O.; Ferrus, R.; Agusti, R. A Robustness Analysis of Learning-Based Coexistence Mechanisms for LTE-U Operation in Non-Stationary Conditions. In Proceedings of the IEEE Vehicular Technology Conference (VTC Fall), Boston, MA, USA, 6-9 September 2015; pp. 1-5.

47. Pérez-Romero, J.; Sallent, O.; Ahmadi, H.; Macaluso, I. On modeling channel selection in LTE-U as a repeated game. In Proceedings of the 2016 IEEE Wireless Communications and Networking Conference, Doha, Qatar, 3-6 April 2016; pp. 1-6

48. Guan, Z.; Melodia, T. CU-LTE: Spectrally-efficient and fair coexistence between LTE and Wi-Fi in unlicensed bands. In Proceedings of the IEEE INFOCOM 2016-The 35th Annual IEEE International Conference on Computer Communications, San Francisco, CA, USA, 10-15 April 2016; pp. 1-9.

49. Yin, W.; Wang, J. Small cell deployment in licensed/unlicensed band: A local interaction game framework. In Proceedings of the International Conference on Wireless Communications \& Signal Processing (WCSP), Nanjing, China, 15-17 October 2015; pp. 1-5.

50. Chen, M.; Saad, W.; Yin, C. Echo State Networks for Self-Organizing Resource Allocation in LTE-U with Uplink-Downlink Decoupling. IEEE Trans. Wirel. Commun. 2017, 16, 3-16. [CrossRef]

51. Cai, F.; Gao, Y.; Cheng, L.; Sang, L.; Yang, D. Spectrum sharing for LTE and WiFi coexistence using decision tree and game theory. In Proceedings of the 2016 IEEE Wireless Communications and Networking Conference, Doha, Qatar, 3-6 April 2016; pp. 1-6.

52. Challita, U.; Dong, L.; Saad, W. Proactive Resource Management for LTE in Unlicensed Spectrum: A Deep Learning Perspective. IEEE Trans. Wirel. Commun. 2018, 17, 4674-4689. [CrossRef]

53. Lan, Y.; Wang, L.; Jiang, H.; Takeda, K.; Harada, H.; Wenfang, T.; Nagata, S. A Field Trial of Unlicensed LTE (U-LTE) in $5.8 \mathrm{GHz}$ Band. In Proceedings of the IEEE Vehicular Technology Conference (VTC Fall), Boston, MA, USA, 6-9 September 2015; pp. 1-5.

54. Fang, Y.; Wang, C.; Ding, G.; Sun, Y. Optimal traffic balancing scheme in small cells between licensed and unlicensed bands. In Proceedings of the International Conference on Wireless Communications \& Signal Processing, Nanjing, China, 15-17 October 2015; pp. 1-6.

55. Xu, Y.; Yin, R.; Chen, Q.; Yu, G. Joint licensed and unlicensed spectrum allocation for unlicensed LTE. In Proceedings of the IEEE Annual International Symposium on Personal, Indoor, and Mobile Radio Communications (PIMRC), Hong Kong, China, 30 August-2 September 2015; pp. 1912-1917. 
56. Jung, H.; Um, J.; Yoo, S.; Park, S. Throughput enhancement with carrier selection for LTE in unlicensed band. In Proceedings of the International Conference on Information and Communication Technology Convergence (ICTC), Jeju, Korea, 28-30 October 2015; pp. 837-839.

57. Hillery, W.J.; Mangalvedhe, N.; Bartlett, R.; Huang, Z.; Kovacs, I.Z. A Network Performance Study of LTE in Unlicensed Spectrum. In Proceedings of the IEEE Globecom Workshops (GC Wkshps), San Diego, CA, USA, 6-10 December 2015; pp. 1-7.

58. Song, H.; Fang, X. A spectrum etiquette protocol and interference coordination for LTE in unlicensed bands (LTE-U). In Proceedings of the IEEE International Conference on Communication Workshop (ICCW), London, UK, 8-12 June 2015; pp. 2338-2343.

59. Li, Z.; Dong, C.; Li, A.; Wang, H. Traffic offloading from LTE-U to WiFi: A multi-objective optimization approach. In Proceedings of the 2016 IEEE International Conference on Communication Systems (ICCS), Shenzhen, China, 14-16 December 2016; pp. 1-5.

60. Voicu, A.M.; Simic, L.; Petrova, M. Coexistence of pico- and femto-cellular LTE-unlicensed with legacy indoor Wi-Fi deployments. In Proceedings of the IEEE International Conference on Communication Workshop (ICCW), London, UK, 8-12 June 2015; pp. 2294-2300.

61. Ko, H.; Lee, J.; Pack, S. A Fair Listen-Before-Talk Algorithm for Coexistence of LTE-U and WLAN. IEEE Trans. Veh. Technol. 2016, 65, 10116-10120. [CrossRef]

62. Yin, R.; Yu, G.; Maaref, A.; Li, G.Y. Adaptive LBT for Licensed Assisted Access LTE Networks. In Proceedings of the IEEE Global Communications Conference (GLOBECOM), San Diego, CA, USA, 6-10 December 2015; pp. 1-6.

63. Ding, W.; Wen, X.; Lu, Z.; Jing, W.; Zeng, Y. A fair listen-before-talk mechanism for LTE-U using a coalition formation approach. In Proceedings of the 2016 International Symposium on Wireless Communication Systems (ISWCS), Poznan, Poland, 20-23 September 2016; pp. 486-490.

64. Bhorkar, A.; Ibars, C.; Zong, P. Performance analysis of LTE and Wi-Fi in unlicensed band using stochastic geometry. In Proceedings of the IEEE Annual International Symposium on Personal, Indoor, and Mobile Radio Communication (PIMRC), Washington, DC, USA, 2-5 September 2014; pp. 1310-1314.

65. Li, Y.; Li, Q.; Zheng, J. Performance Analysis of Licensed-Assisted Access Using LTE with Adaptive Carrier Selection. In Proceedings of the IEEE Globecom Workshops (GC Wkshps), San Diego, CA, USA, 6-10 December 2015; pp. 1-6.

66. Wang, X.; Quek, T.Q.S.; Sheng, M.; Li, J. Throughput and Fairness Analysis of Wi-Fi and LTE-U in Unlicensed Band. IEEE J. Sel. Areas Commun. 2017, 35, 63-78. [CrossRef]

67. Chen, C.; Ratasuk, R.; Ghosh, A. Downlink Performance Analysis of LTE and WiFi Coexistence in Unlicensed Bands with a Simple Listen-Before-Talk Scheme. In Proceedings of the IEEE Vehicular Technology Conference (VTC Spring), Glasgow, UK, 11-14 May 2015; pp. 1-5.

68. Dama, S.; Kumar, A.; Kuchi, K. Performance Evaluation of LAA-LBT Based LTE and WLAN's Co-Existence in Unlicensed Spectrum. In Proceedings of the IEEE Globecom Workshops (GC Wkshps), San Diego, CA, USA, 6-10 December 2015; pp. 1-6.

69. Kini, A.V.; Canonne-Velasquez, L.; Hosseinian, M.; Rudolf, M.; Stern-Berkowitz, J. Wi-Fi-LAA coexistence: Design and evaluation of Listen Before Talk for LAA. In Proceedings of the Annual Conference on Information Science and Systems (CISS), Princeton, NJ, USA, 16-18 March 2016; pp. 157-162.

70. Li, Y.; Zheng, J.; Li, Q. Enhanced listen-before-talk scheme for frequency reuse of licensed-assisted access using LTE. In Proceedings of the IEEE International Symposium on Personal, Indoor, and Mobile Radio Communications (PIMRC), Hong Kong, China, 30 August-2 September 2015; pp. 1918-1923.

71. Tao, T.; Han, F.; Liu, Y. Enhanced LBT algorithm for LTE-LAA in unlicensed band. In Proceedings of the IEEE International Symposium on Personal, Indoor, and Mobile Radio Communications (PIMRC), Hong Kong, China, 30 August-2 September 2015; pp. 1907-1911.

72. Zhang, M.; Zhang, X.; Chang, Y.; Yang, D. Dynamic Uplink Radio Access Selection of LTE Licensed-Assisted Access to Unlicensed Spectrum: An Optimization Game. IEEE Commun. Lett. 2016, 20, 2510-2513. [CrossRef]

73. Cano, C.; Leith, D.J. Unlicensed LTE/WiFi coexistence: Is LBT inherently fairer than CSAT? In Proceedings of the 2016 IEEE International Conference on Communications (ICC), Kuala Lumpur, Malaysia, 23-27 May 2016; pp. 1-6.

74. Bairagi, A.K.; Tran, N.H.; Saad, W.; Han, Z.; Hong, C.S. A Game-Theoretic Approach for Fair Coexistence Between LTE-U and Wi-Fi Systems. IEEE Trans. Veh. Technol. 2019, 68, 442-455. [CrossRef] 
75. Galanopoulos, A.; Tsiftsis, T.; Foukalas, F. Licensed Assisted Access: Key enabling functionalities and initial results. In Proceedings of the International Symposium on Wireless Communication Systems (ISWCS), Brussels, Belgium, 25-28 August 2015; pp. 171-175.

76. Li, Y.; Baccelli, F.; Andrews, J.G.; Novlan, T.D.; Zhang, J. Modeling and Analyzing the Coexistence of Licensed-Assisted Access LTE and Wi-Fi. In Proceedings of the IEEE Globecom Workshops (GC Wkshps), San Diego, CA, USA, 6-10 December 2015; pp. 1-6.

77. Li, J.; Han, Y. Multi-RAT wireless network capacity optimization under optimal spectrum splitting in LTE-U. In Proceedings of the 2016 IEEE Wireless Communications and Networking Conference, Doha, Qatar, 3-6 April 2016; pp. 1-6.

78. Li, Z.; Liu, C.; Li, H.; Li, Z. A Field Trial of Wi-Fi Co-Existing with U-LTE Based on Cell On/Off on Unlicensed Spectrum. In Proceedings of the 2016 IEEE 83rd Vehicular Technology Conference (VTC Spring), Nanjing, China, 15-18 May 2016; pp. 1-5.

79. Li, J.; He, W.; Han, Y. HetNet Capacity Optimization under Optimal Femtocell Operation in LTE-U. In Proceedings of the 2016 IEEE 83rd Vehicular Technology Conference (VTC Spring), Nanjing, China, 15-18 May 2016; pp. 1-5.

80. Haile, B.B.; Mutafungwa, E.; Hämäläinen, J. LTE-Advanced enhancements for self-backhauled LTE-U small cells: An Addis Ababa case study. In Proceedings of the AFRICON 2015, Addis Ababa, Ethiopia, 14-17 September 2015; pp. 1-5.

81. Lien, S.Y.; Cheng, S.M.; Chen, K.C.; Kim, D.I. Resource-Optimal Licensed-Assisted Access in Heterogeneous Cloud Radio Access Networks with Heterogeneous Carrier Communications. IEEE Trans. Veh. Technol. 2016, 65, 9915-9930. [CrossRef]

82. Zhang, R.; Wang, M.; Cai, L.X.; Shen, X.; Xie, L.L.; Cheng, Y. Modeling and Analysis of MAC Protocol for LTE-U Co-Existing with Wi-Fi. In Proceedings of the IEEE Global Communications Conference (GLOBECOM), San Diego, CA, USA, 6-10 December 2015; pp. 1-6.

83. Bhorkar, A.; Ibars, C.; Zong, P. On the throughput analysis of LTE and WiFi in unlicensed band. In Proceedings of the Asilomar Conference on Signals, Systems and Computers, Pacific Grove, CA, USA, 2-5 November 2014; pp. 1309-1313.

84. Bhorkar, A.; Ibars, C.; Papathanassiou, A.; Zong, P. Medium access design for LTE in unlicensed band. In Proceedings of the IEEE Wireless Communications and Networking Conference Workshops (WCNCW), New Orleans, LA, USA, 9-12 March 2015; pp. 369-373.

85. Xu, J.; Sun, Y.; Ji, Y.; Tian, Y. A Time and Frequency Division Algorithm for Control Signaling Collision Avoidance in Standalone LTE-unlicensed Networks. In Proceedings of the 2016 International Conference on Networking and Network Applications (NaNA), Hakodate, Japan, 23-25 July 2016; pp. 12-16.

86. Kwan, R.; Pazhyannur, R.; Seymour, J.; Chandrasekhar, V.; Saunders, S.R.; Bevan, D.; Konstantinou, K. Fair co-existence of Licensed Assisted Access LTE (LAA-LTE) and Wi-Fi in unlicensed spectrum. In Proceedings of the Computer Science and Electronic Engineering Conference (CEEC), Colchester, UK, 24-25 September 2015; pp. 13-18.

87. Lee, J.; Ko, H.; Pack, S. Performance evaluation of LTE-unlicensed in handover scenarios. In Proceedings of the International Conference on Information and Communication Technology Convergence (ICTC), Jeju, Korea, 28-30 October 2015; pp. 1043-1045.

88. Choi, S.; Park, S. Co-existence analysis of duty cycle method with Wi-Fi in unlicensed bands. In Proceedings of the International Conference on Information and Communication Technology Convergence (ICTC), Jeju, Korea, 28-30 October 2015; pp. 894-897.

89. Choi, S.; Park, S. Performance analysis of various co-existence methods with Wi-Fi in unlicensed bands. In Proceedings of the International Conference on Information and Communication Technology Convergence (ICTC), Jeju, Korea, 28-30 October 2015; pp. 729-732.

90. Tao, R.; Li, L.; Chu, X.; Zhang, J. Handover mechanism and performance evaluation for LTE-LAA systems. In Proceedings of the 2016 IEEE 17th International Workshop on Signal Processing Advances in Wireless Communications (SPAWC), Edinburgh, UK, 3-6 July 2016; pp. 1-5.

91. 3GPP Commits to 5G NR in Unlicensed Spectrum in Its Next Release. Available online: https://www. qualcomm.com/news/onq/2018/12/13/3gpp-commits-5g-nr-unlicensed-spectrum-its-next-release (accessed on 13 December 2018).

92. Gozalvez, J. 5G Worldwide Developments [Mobile Radio]. IEEE Veh. Technol. Mag. 2017, 12, 4-11. [CrossRef] 
93. Lien, S.; Shieh, S.; Huang, Y.; Su, B.; Hsu, Y.; Wei, H. 5G New Radio: Waveform, Frame Structure, Multiple Access, and Initial Access. IEEE Commun. Mag. 2017, 55, 64-71. [CrossRef]

94. Soret, B.; Domenico, A.D.; Bazzi, S.; Mahmood, N.H.; Pedersen, K.I. Interference Coordination for 5G New Radio. IEEE Wirel. Commun. 2018, 25, 131-137. [CrossRef]

95. Dahlman, E.; Parkvall, S. NR-The New 5G Radio-Access Technology. In Proceedings of the IEEE Vehicular Technology Conference (VTC Spring), Porto, Portugal, 3-6 June 2018; pp. 1-6.

96. Richardson, T.; Kudekar, S. Design of Low-Density Parity Check Codes for 5G New Radio. IEEE Commun. Mag. 2018, 56, 28-34. [CrossRef]

97. Onggosanusi, E.; Rahman, M.S.; Guo, L.; Kwak, Y.; Noh, H.; Kim, Y.; Chen, R. Modular and High-Resolution Channel State Information and Beam Management for 5G New Radio. IEEE Commun. Mag. 2018, 56, 48-55. [CrossRef]

98. Zhang, J.; Zheng, Z.; Zhang, Y.; Xi, J.; Zhao, X.; Gui, G. 3D MIMO for 5G NR: Several Observations from 32 to Massive 256 Antennas Based on Channel Measurement. IEEE Commun. Mag. 2018, 56, 62-70. [CrossRef]

99. Park, H.; Lee, Y.; Kim, T.; Kim, B.; Lee, J. Handover Mechanism in NR for Ultra-Reliable Low-Latency Communications. IEEE Netw. 2018, 32, 41-47. [CrossRef]

100. Patzold, M. Countdown for the Full-Scale Development of 5G New Radio [Mobile Radio]. IEEE Veh. Technol. Mag. 2018, 13, 7-13. [CrossRef]

101. Xiao, Y.; Hirzallah, M.; Krunz, M. Distributed Resource Allocation for Network Slicing Over Licensed and Unlicensed Bands. IEEE J. Sel. Areas Commun. 2018, 36, 2260-2274. [CrossRef]

102. Wang, P.; Di, B.; Zhang, H.; Bian, K.; Song, L. Cellular V2X Communications in Unlicensed Spectrum: Harmonious Coexistence with VANET in 5G Systems. IEEE Trans. Wirel. Commun. 2018, 17, 5212-5224. [CrossRef]

103. Bairagi, A.K.; Abedin, S.F.; Tran, N.H.; Niyato, D.; Hong, C.S. QoE-Enabled Unlicensed Spectrum Sharing in 5G: A Game-Theoretic Approach. IEEE Access 2018, 6, 50538-50554. [CrossRef]

104. MulteFire Release 1.1 Technical Overview White Pape. Available online: www.multefire.org (accessed on 16 July 2020).

105. Karvonen, H.; Hämäläinen, M.; Iinatti, J.; Pomalaza-Ráez, C. Coexistence of wireless technologies in medical scenarios. In Proceedings of the European Conference on Networks and Communications (EuCNC), Oulu, Finland, 12-15 June 2017; pp. 1-5.

106. Hosein, P.; Seok, W.; Kwak, J.S.; Ni, A. Emerging network technologies and network neutrality conformance. In Proceedings of the 2017 19th Asia-Pacific Network Operations and Management Symposium (APNOMS), Seoul, Korea, 27-29 September 2017; pp. 31-36.

107. Schrader, V.; Vilgelm, M.; Kellerer, W. On Random Access Channel Performance and M2M Support in Standalone LTE Unlicensed. In Proceedings of the IEEE Global Communications Conference, Singapore, 4-8 December 2017; pp. 1-7.

108. Bitar, N.; al Kalaa, M.O.; Seidman, S.J.; Refai, H.H. On the Coexistence of LTE-LAA in the Unlicensed Band: Modeling and Performance Analysis. IEEE Access 2018, 6, 52668-52681. [CrossRef]

109. Ismaiel, B.; Abolhasan, M.; Smith, D.; Ni, W.; Franklin, D. A Survey and Comparison of Device-to-Device Architecture Using LTE Unlicensed Band. In Proceedings of the IEEE Vehicular Technology Conference (VTC Spring), Sydney, NSW, Australia, 4-7 June 2017; pp. 1-5.

110. Labib, M.; Marojevic, V.; Reed, J.H.; Zaghloul, A.I. Extending LTE into the Unlicensed Spectrum: Technical Analysis of the Proposed Variants. IEEE Commun. Stand. Mag. 2017, 1, 31-39. [CrossRef]

111. Rosa, C.; Kuusela, M.; Frederiksen, F.; Pedersen, K.I. Standalone LTE in Unlicensed Spectrum: Radio Challenges, Solutions, and Performance of MulteFire. IEEE Commun. Mag. 2018, 56, 170-177. [CrossRef]

112. Do-Yun, K.; Choi, Y.J. Spectrum coordination of multi-RAT D2D for IoT service. In Proceedings of the International Conference on Information and Communication Technology Convergence (ICTC), Jeju, Korea, 28-30 October 2015; pp. 671-673.

(C) 2020 by the authors. Licensee MDPI, Basel, Switzerland. This article is an open access article distributed under the terms and conditions of the Creative Commons Attribution (CC BY) license (http:/ / creativecommons.org/licenses/by/4.0/). 